\title{
Self-Sustained Conditions in Inhomogeneous Fields
}

\author{
B. Held $\left({ }^{1, *}\right)$, N. Soulem $\left({ }^{1}\right)$, R. Peyrous $\left({ }^{1}\right)$ and N. Spyrou $\left({ }^{2}\right)$ \\ ( ${ }^{1}$ Laboratoire d'Électronique, des Gaz et des Plasmas, Unıversité de Pau, 64000 Pau, France \\ $\left({ }^{2}\right)$ Electrotechnic Materials Laboratory, Patras University, 26500 Patras-Rio, Greece
}

(Received 12 May 1997, revised 24 June 1997, accepted 2 July 1997)

PACS.51.50.+v - Electrical properties (ionization, breakdown, electron and ion mobility, etc.) PACS.52 80.Hc - Glow; corona

\begin{abstract}
A theoretical model allowing to plot the breakdown voltage $V_{\mathrm{B}}$ as a function of the product $p d$ (pressure $\times$ interelectrode distance) is presented for a D.C corona discharge. For a parabolic point, it is shown that the Paschen curve can be generalized to inhomogeneous fields, since the homogeneous fields are the asymptotic limit of this theory. The analytical expression obtained from this model is finally plotted and compared to experimental results in nitrogen and dry air.
\end{abstract}

Résumé. - Un modèle théorıque permettant de tracer le potentiel disruptif $V_{\mathrm{B}}$ en fonction du produit $p d$ (pression $\times$ distance interélectrodes) est présenté pour une décharge couronne en tension contınue. On montre, pour une pointe parabolique, que la corbe de Paschen peut être généralisée au cas des champs inhomogènes, le cas du champ homogène étant la limite asymptotique de cette théorie. l'expression analytique obtenue à partir de ce modèle est finalement tracée et comparée aux résultats expérimentaux obtenus dans l'air et l'azote.

\section{Introduction}

For homogeneous fields (parallel-plates gap), the conditions for a self-sustained discharge has been well known for many years $[1,4]$ In this case, the breakdown voltage $V_{\mathrm{B}}$ is deduced from the breakdown equation as a function of the gap distance and the pressure, by introducing the first ionization coefficient $\alpha$, the attachment coefficient $\eta$ (for electronegative gases) and the secondary ionization coefficient $\gamma . \alpha$ and $\eta$ are generally well documented for many gases, while informations on $\gamma$ are hardly available, depending on gases, surface materials, ... [4,5]. Consequently, some discrepancies between theory and experiments abserved for the Paschen curve are mainly related to the uncertainties on the effective secondary coefficient $\gamma$. Paschen's curve is a quantitative expression of simlarity law. The breakdown potential $V_{\mathrm{B}}$ is a function of the product ( $p$ refers to the gas pressure and $d$ to the gap length) which is a measure of the number of collisions an electron makes by crossing the gap. As long as this number remains

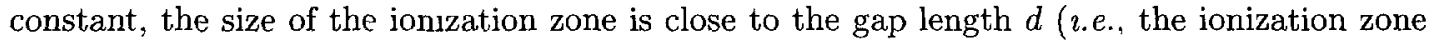
occupies the whole gap). A self-sustained discharge occurs when the electron regeneration is ensured by $\gamma$ cathode feedback mechanism and not by external ionization agent.

$\left({ }^{*}\right)$ Author for correspondence

(C) Les Éditions de Physıque 1997 
For inhomogeneous fields (point-to-plane gap), the difficulty increases because the coefficients $\alpha, \eta$, depending on the field/pressure ratio $(E / p)$, are not constant in the interelectrode gap. Furthermore, different microscopic mechanisms occur: they result from collisions between the background free electrons (produced by cosmic rays) and neutral species, and modify the total number of free charges. As a consequence, a local space-charge field is produced in a limited ionization region; the local electric field then results from the superposition of the geometric electric field $\mathbf{E}_{\mathrm{G}}$ and the space-charge field $\mathbf{E}_{\mathrm{C}}$. In these conditions, the size of the ionization region is not constant with space and time. Consequently, the ionization coefficient $\gamma$, which is relevant to the secondary electrons coming from the discharge zone outside the limited ionization region, cannot be easily defined.

In spite of these difficulties, $V$ vs. pd plots were deduced from experiments performed many years ago in a wire-to-cylinder geometry [2,6-8]. Paschen-like curves are also observed $[9,10]$ in a point-to-plane geometry. This paper shows that it is possible to deduced some general results by comparing symmetrical (parallel-plate gap) and dissymmetrical (point-to-plane gap) discharges.

In the first part of this paper, a theoretical model is presented and Paschen-like curves are deduced. The second part is devoted to experimental results obtained in nitrogen and air (2.e. without significant space charge effect). In the third part, the theoretical and experimental results are compared and analysed.

\section{Theory}

The observation of a $V-I$ characteristic relation for a common gap shows two important regions separated by an abrupt voltage transition. The current is given by the general expression:

$$
I=M I_{0}
$$

where $I_{0}$ is the current of the primary electrons and $M$ the factor of multiplication.

2.1. Homogeneous Field. - For homogeneous fields (parallel-plates gap), the first region corresponds to the Townsend discharge with an overexponential multiplication of charges. The abrupt transition is reached for the breakdown potential $V_{\mathrm{B}}$, when the discharge becomes independent on the external sources of ionization. In this case (symmetrical discharge) and for steady state conditions, the factor $M$ is given [3] by:

$$
M=\frac{\bar{\alpha}+\alpha[\exp (\bar{\alpha} d)-1]}{\bar{\alpha}-\alpha \gamma[\exp (\bar{\alpha} d)-1]}
$$

where $\bar{\alpha}$ is the effective first ionization coefficient defined by:

$$
\bar{\alpha}=\alpha-\eta \text {. }
$$

The breakdown criterion is reached when the denominator of (2) equals zero:

$$
\bar{\alpha}-\alpha \gamma[\exp (\bar{\alpha} d)-1]=0 .
$$

For a non electronegative gas $(\eta=0)$, expression (4) is reduced to (15):

$$
1-\gamma[\exp (\alpha d)-1]=0 \text {. }
$$

By introducing the first ionization coefficient $[2-4,11-13]$ :

$$
\frac{\alpha}{p}=A \exp (-B p / E)
$$


we obtain the well known Paschen law [1-4]:

$$
V_{\mathrm{B}}=B \frac{(p d)}{\ln \left(\frac{A}{\ln (1+1 / \gamma)}\right)+\ln (p d)} .
$$

The minimum is given by:

$$
\left(V_{\mathrm{B}}\right)_{\mathrm{m}}=B(p d)_{\mathrm{m}}
$$

and:

$$
(p d)_{\mathrm{m}}=\frac{\vec{e}}{A} \ln (1+1 / \gamma)
$$

where $\bar{e}$ is the natural logarithm number.

The coefficients $A$ and $B$ are well known for many gases [2-4]: we can then deduce $\gamma$ at the minimum of Paschen, from equation (9):

$$
\gamma_{\mathrm{m}}=\frac{1}{\left[\exp \left[A(p d)_{\mathrm{m}} / \bar{e}\right]-1\right]} .
$$

These results are generally in good agreement with the experiments; the discrepancies mainly result from the uncertainties on the secondary emission coefficient $\gamma$.

2.2. Inhomogeneous Fields. - For inhomogeneous fields (point-to-plane gap), the first regime can be interpreted as a Townsend-like discharge. The factor $M(2)$ becomes $[5,14-17]$ in the steady state assumption:

$$
M=\frac{1+\int_{0}^{d} \alpha \exp \left(\int_{0}^{z} \bar{\alpha} \mathrm{d} z^{\prime}\right) \mathrm{d} z}{1-\gamma \int_{0}^{d} \alpha \exp \left(\int_{0}^{z} \bar{\alpha} \mathrm{d} z^{\prime}\right) \mathrm{d} z}
$$

$\gamma$ is then interpreted as an average on several elementary mechanisms (in the interelectrode gap) for secondary electrons generation.

Then, the breakdown criterion can be written:

$$
1-\gamma \int_{0}^{d} \alpha \exp \left(\int_{0}^{z} \bar{\alpha} \mathrm{d} z^{\prime}\right) \mathrm{d} z=0
$$

where $d$ refers to the point to plane distance.

For a non electronegative gas $(\eta=0)$, expression (12) becomes (13):

$$
1-\gamma \int_{0}^{d} \alpha \exp \left(\int_{0}^{z} \alpha \mathrm{d} z^{\prime}\right) \mathrm{d} z=0 .
$$

The first ionization coefficient $\alpha$ can be expressed by introducing the geometric electric field. For a parabolic point of radius $R$, at the distance $z$ from the plane, the electric field is given [6] by:

$$
E(z)=\frac{V}{(d+R / 2-z) \ln (2 d / R+1)} .
$$

In this condition, $\alpha(6)$ can be written:

$$
\alpha=\alpha_{0} \exp (C z / d)
$$


with:

$$
\begin{aligned}
\alpha_{0} & =A p \exp [-C(1+R / 2 d)] \\
C & =B p d / V^{\prime}
\end{aligned}
$$

and:

$$
V^{\prime}=\frac{V}{\ln (2 d / R+1)}
$$

By introducing $\alpha$ (15) in equation (13), we find.

$$
\frac{\alpha_{0} d}{C}[\exp (C)-1]=\ln \left(1+\frac{1}{\gamma}\right)
$$

and finally, using (16) and (17).

$$
V_{\mathrm{B}}^{\prime} \exp \left(-B \frac{R}{2 d} \frac{p d}{V_{\mathrm{B}}^{\prime}}\right)\left(1-\exp \left(-B \frac{p d}{V_{\mathrm{B}}^{\prime}}\right)\right)=\frac{B}{A} \ln (1+1 / \gamma) .
$$

By deriving this expression relatively to $p d$, we obtain the minimum:

$$
\left(V_{\mathrm{B}}\right)_{\mathrm{m}}=B(p d)_{\mathrm{m}}
$$

with:

$$
(p d)_{\mathrm{m}}=\frac{F(R, d)}{A} \ln (1+1 / \gamma)
$$

where the geometrical factor $F(R, d)$ given by (23).

$$
F(R, d)=\left(\frac{R}{2 d}+1\right) \ln \left(\frac{2 d}{R}+1\right) \exp \left(\frac{R}{2 d} \ln \left(\frac{2 d}{R}+1\right)\right)
$$

depends on the radius $R$ of the point and on the point-to-plane distance $d$. In the asymptotic limit $(R / 2 d \gg 1)$, we find from equation $(20)$ :

$$
p d \exp \left(-B \frac{p d}{V_{\mathrm{B}}}\right)=\frac{1}{A} \ln \left(1+\frac{1}{\gamma}\right)
$$

and from equation (23):

$$
F(R, d)=\bar{e}
$$

2.e. the general Paschen law for homogeneous fields (Eqs. (7) and (9))

In the case of an inhomogeneous field, it is also possible to deduce $\gamma_{\mathrm{m}}$ at the minimum of the plot $V$ vs. (pd), using equation (22):

$$
\gamma_{\mathrm{m}}=\frac{1}{\left[\exp \left[A(p d)_{\mathrm{m}} / F(R, d)\right]-1\right]} .
$$

We obtain the Paschen-like law (20) assuming that the secondary ionization coefficient $\gamma$ takes a constant mean value and that the gas is non electronegative. Furthermore, the geometrical electric field is not constant in the interelectrode gap (14), that is when $z$ varies from zero to $d$. In these conditions, the analytic expression for the first ionization coefficient $\alpha(6)$ must be known in a wide range of ratio $E / p$. In addition, the Paschen-like law is not fundamentally modified for an electronegative gas, since we have only to take care of the attachment in the weaker field regions ( .e. near the plane $z \cong 0$ ). In this case, the first ionization coefficient $\alpha$ 


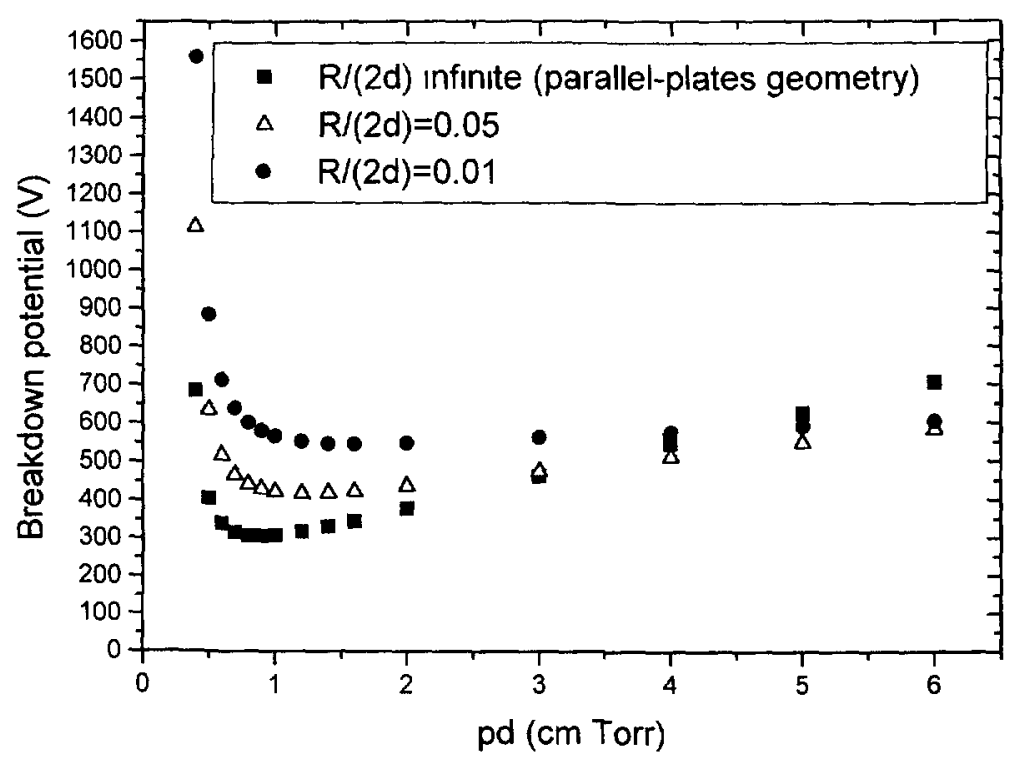

Fig. 1. - Comparison between the $V_{\mathrm{B}}=f(p d)$ theoretical curves in homogeneous and inhomogeneous fields in nitrogen $(\gamma=0.02)$.

and the attachment coefficient $\eta$ take very small values $\left(\alpha / p, \eta / p \leq p \leq 10^{-2} \mathrm{~cm}^{-1} \operatorname{Torr}^{-1}\right)$ (1mbar $=0.75$ Torr) in oxygen and in air [13] and $\int_{0}^{z} \bar{\alpha} \mathrm{d} z^{\prime}$ (Eq. (12)) is not significantly changed when $\bar{\alpha}$ is replaced by $\alpha$ (Eq. (15)). Keeping in mind these approximations, we apply this theory to nitrogen and air.

The theoretical results are presented in Figures 1 and 2 where the parallel-plates and pointto-plane cases are compared. For each application, the parameters $A, B$ of the first ionization coefficient $\alpha(6)$ and the secondary ionization coefficient $\gamma$ are introduced in equation (20), with the data given in [4] (i.e. $A=12 \mathrm{~cm}^{-1}$ Torr ${ }^{-1}, B=342 \mathrm{~V} \mathrm{~cm}^{-1} \mathrm{Torr}^{-1}, \gamma=2 \times 10^{-2}$ for nitrogen and $A=15 \mathrm{~cm}^{-1} \operatorname{Torr}^{-1}, B=365 \mathrm{~V} \mathrm{~cm}^{-1}$ Torr $^{-1}, \gamma=10^{-2}$ for air) (1 mbar $=0.75$ Torr). Figures 1 (nitrogen) and 2 (air), show that the minimum breakdown potential $\left(V_{\mathrm{B}}\right)_{\mathrm{m}}$ and the corresponding $(p d)_{\mathrm{m}}$ values increase with the inhomogeneity of the field, that is when the ratio $R / 2 d$ decreases from infinite (parallel-plates case) to small values (the point-to-plane case is given for $R / 2 d=0.05$ and 0.01 ). Furthermore, the slopes of the plots decrease for $(p d)$ values higher than the minimum $(p d)_{\mathrm{m}}$. These results must be interpreted by keeping in mind that the secondary ionization coefficient $\gamma$ has not the same signification (neither the same value) in the parallel-plates and in the point-to-plane cases (defined as the corresponding number of secondary cathode electrons produced by ionic bombardment for a parallel-plates gap and relative to the electrons produced in the volume surrounding the head of the streamer for a point-to-plane gap). Furthermore, this model is only valid for extremely low currents $(1 \mu \mathrm{A})$, since the electric field distribution not being significantly disturbed by space charge effects in this case. 


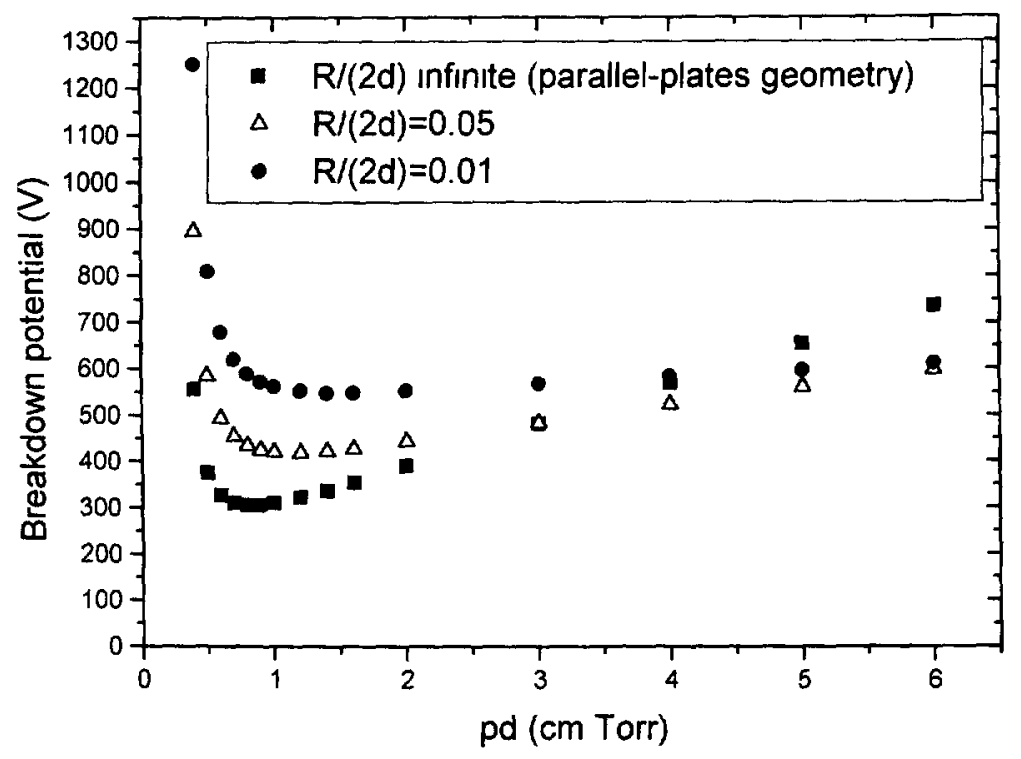

Fig. 2 - Comparison between the $V_{\mathrm{B}}=f(p d)$ theoretical curves in homogeneous and inhomogeneous fields in air $(\gamma=0.01)$.

\section{Experiments}

3.1. Experimental Set-UP and Conditions. - Figure 3 shows that the discharge is generated in a stainless-steel cell either between two parallel plates or in a point-to-plane device. The point and the plates are stainless-steel too. The curvature radius $R$ of the tip of the rod is $0.1 \mathrm{~cm}$. The insulation of the rod is presented in Figure 4a. It can be seen that the tip of the rod is alone submitted to the discharge, since the rod is totally insulated.

The potential applied to the point is positive in each experiment. The chosen point-toplane distance is $d=1 \mathrm{~cm}$ because the range of the $p d$ products for which breakdown occurs contains $(p d)_{\mathrm{m}}$ value of the corresponding Paschen curve. In other cases, depending on the Paschen curves relative to the various possibilities of breakdown in the cell, the discharge can be initiated over larger distance than $d$ (i.e. between the point and the cell) for the lower values of $p d$ product; so, the Paschen curve obtained is a "composite" one and the minimum breakdown potential $\left(V_{\mathrm{B}}\right)_{\mathrm{m}}$ and its corresponding $(p d)_{\mathrm{m}}$ value are not rigorously defined (experimental results are available in [10]).

Concerning the experiments in homogeneous field, it must be pointed out that the plates are not designed with a Rogowsky profile. To prevent discharge from initiating at the edges of the disks, two insulating rings in PVC was added (Fig. 4b).

Moreover, it was not possible to reduce the pressure below $3 \times 10^{-2} \mathrm{mbar}\left(2.25 \times 10^{-2} \mathrm{Torr}\right)$ before starting the experiment. So, a residual contamination (water, dust) could remain in the cell during the measurements.

The following parts are devoted to the experimental determination of the $V_{\mathrm{B}}=f(p d)$ curves in nitrogen and dry air. Some $V-I$ characteristics are presented, correlated to the current impulses observed using a $200 \mathrm{MHz}$ digital oscllloscope. 


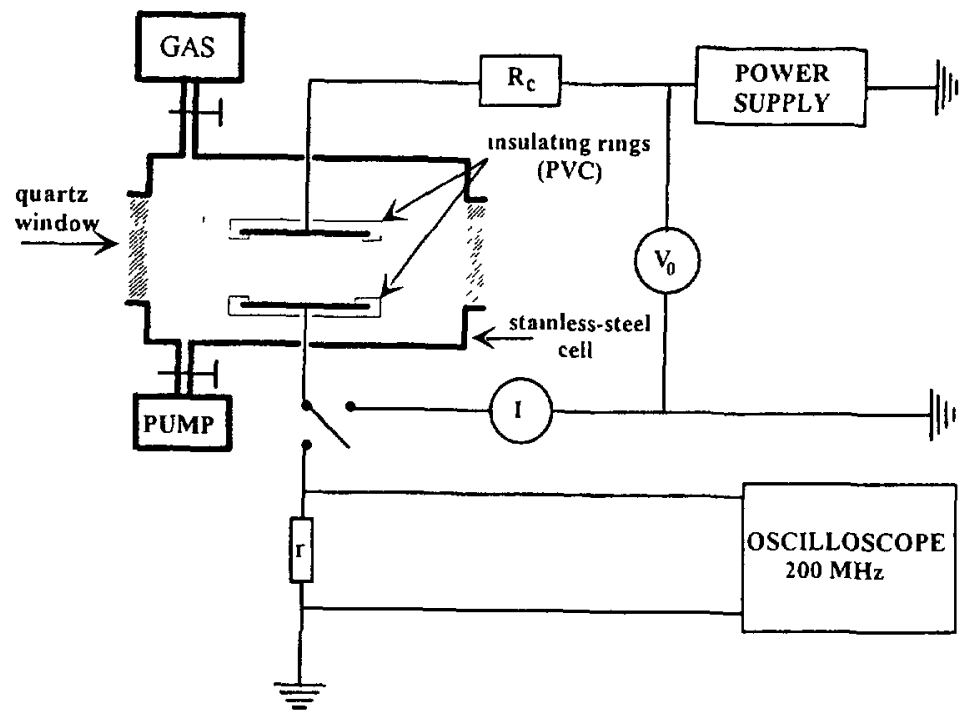

Fig. 3. - Expermental set-up. I: microammeter. $V_{0}$ : voltmeter measuring the power supply voltage. $R_{\mathrm{c}}$ loading impedance.

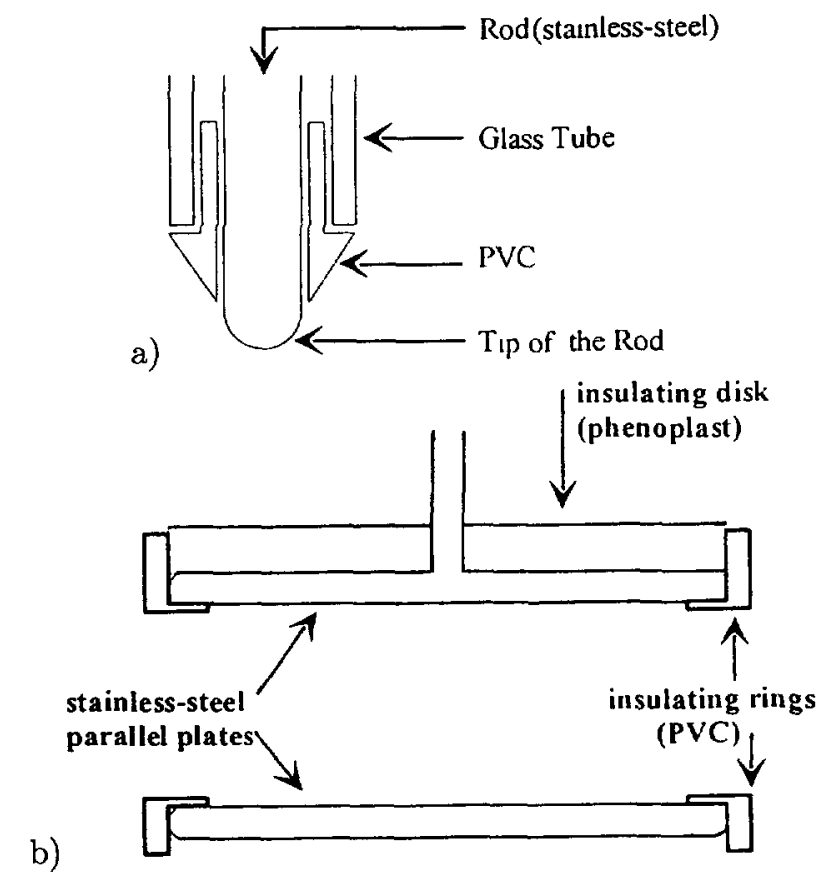

Fig. $4-$ a) insulation of the rod The tip of the rod is alone submitted to the discharge b) insulation of the plates. Two insulating rings in PVC prevent the discharge from initiating at the edges of the plates 


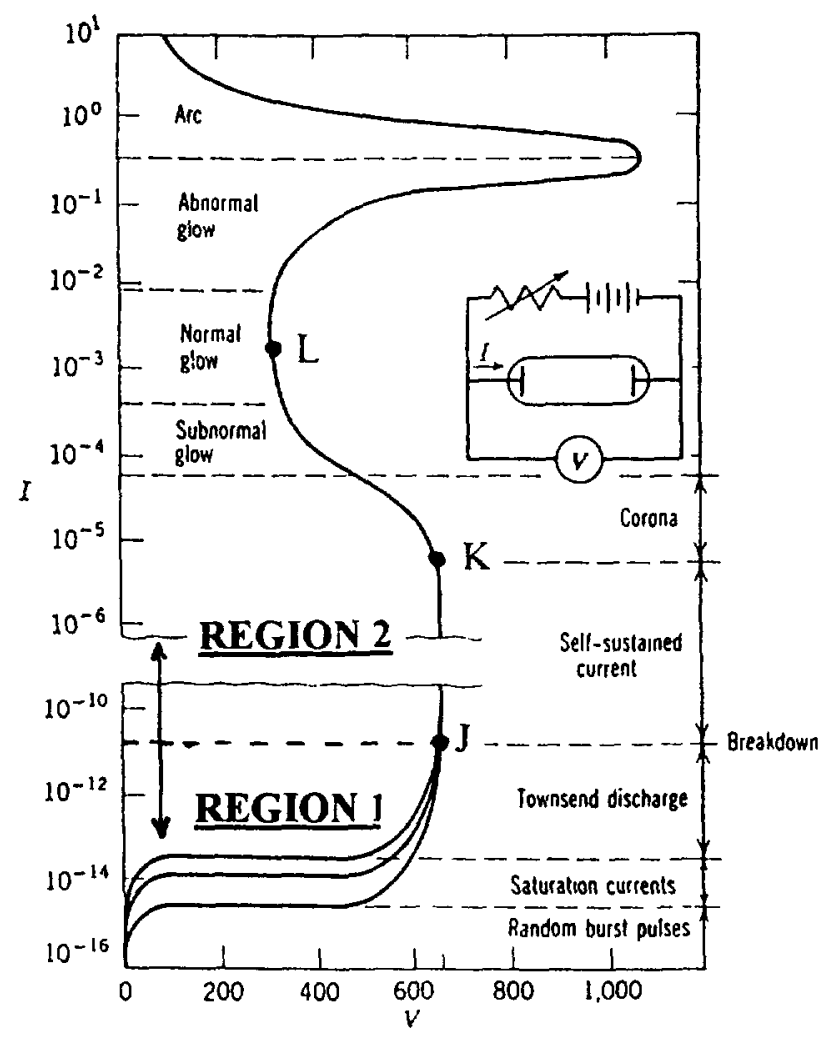

Fig. 5. - Typical voltage-current characteristic in parallel-plates geometry at pressure about 1.33 mbar (1 Torr) (from [3])

3.2. Breakdown Criterion and Particular Points of the Voltage-Current CharACTERISTIC. - The well-known typical voltage-current characteristic obtained in parallelplates device is shown in Figure 5 (from [3]). In region 1, an external ionizing source (UV light, radioactive component) is necessary to produce - and then, to measure - the current $I$ The current is not self-sustained. In region 2, the measured current $I$ is independent of the ionizing source; the current is self-sustamed. The transition to self-sustained discharge is called breakdown and the corresponding interelectrode voltage is known as the breakdown voltage $V_{\mathrm{B}}$.

In our experiment, no external ionizing source is used; thus, no steady current is measured in the region 1 , which is not observed on the $V-I$ characteristics. Figure 6 shows a typical $V-I$ characteristic. Up to the potential $V_{1}$, no current is measured; thus:

$$
V_{1}=V_{0}-R_{\mathrm{c}} I \approx V_{0}
$$

where $V_{1}$ is the interelectrode potential, $V_{0}$ the power supply voltage and $R_{\mathrm{c}}$ the loading impedance $\left(R_{\mathrm{c}}=100 \mathrm{M} \Omega\right)$. An abrupt transition occurs at $V_{1}$, where a steady self-sustained direct current flows in the external circuit followed by the collapse of the interelectrode voltage, thus:

$$
V_{2} \approx V_{1}-R_{\mathrm{c}} I_{2}
$$

Further increase of $V_{0}$ leads to an increase in the current (part JK of the characteristic in Fig. 6). 


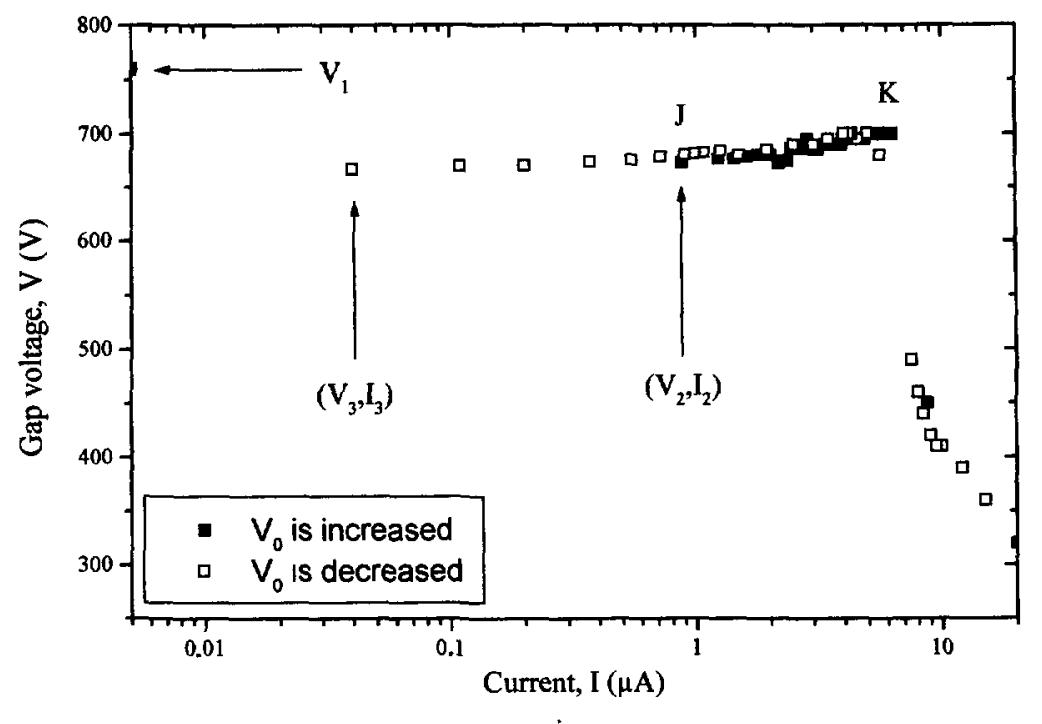

Fig. 6 - Typical $V-I$ characteristic in point-to-plane configuration (nitrogen, $d=1 \mathrm{~cm}$ and $p=5$ $\operatorname{mbar}(3.75$ Torr))

$\left(V_{2}, I_{2}\right)$ being the point of the characteristic at which the discharge becomes self- sustained, one could define $V_{2}$ as the breakdown voltage $V_{\mathrm{B}}$. Nevertheless, when $V_{0}$ is decreased from the point $K$, the current decreases far below $I_{2}$, pointing out an hysteresis. Thus, it is possible to define a potential, $V_{3}$, at which the current vanishes. It will be shown in a next paper that $V_{3}$ corresponds to the breakdown voltage that would be measured if an external ionizing source was used.

The voltage-current characteristics will be discussed in parts 3 and 4 and Paschen curves are presented in part 5.

3.3. Parallel-Plates Geometry: $V$ - I Characteristics. -- The current-voltage characteristic in nitrogen for $p=2,4$ and 8 mbar (1.5, 3 and 6 Torr) and $d=2 \mathrm{~cm}$ is shown in Figure 7.

Over the part $\mathrm{JK}$, the slope $\mathrm{d} V / \mathrm{d} I \approx 0$ and only a direct current flows in the external circuit (see Fig. 8a): it is the self-sustained dark discharge. $\mathrm{d} V / \mathrm{d} I \approx 0$ reveals that the increase in current is not controlled by the space charge, but only by the external circuit ( $V_{0}$ and $R_{\mathrm{c}}$ ). Whatever the pressure, the slope is never positive.

In $\mathrm{K}$, an abrupt transition leads to the glow discharge where $\mathrm{d} V / \mathrm{d} I<0$ (part KL). A recurring state composed of transient current impulses superimposed to the direct component (see Fig. 8b) is established. The current impulses must be related to the periodic formation of the cathodic region, resulting from the development of a critical space charge in the interelectrode gap.

As a conclusion, our experimental current-voltage characteristics are to be compared with the part JKL of the standard one shown in Figure 5.

\subsection{Point-to-Plane Geometry. $V-I$ Characteristics}

3.4 1. Breakdown in Natrogen. - An example of the point-to-plane current-voltage characteristic is shown in Figure 9a for $p=3 \mathrm{mbar}$ (2.25 Torr). On the first part of the curve (JK), 


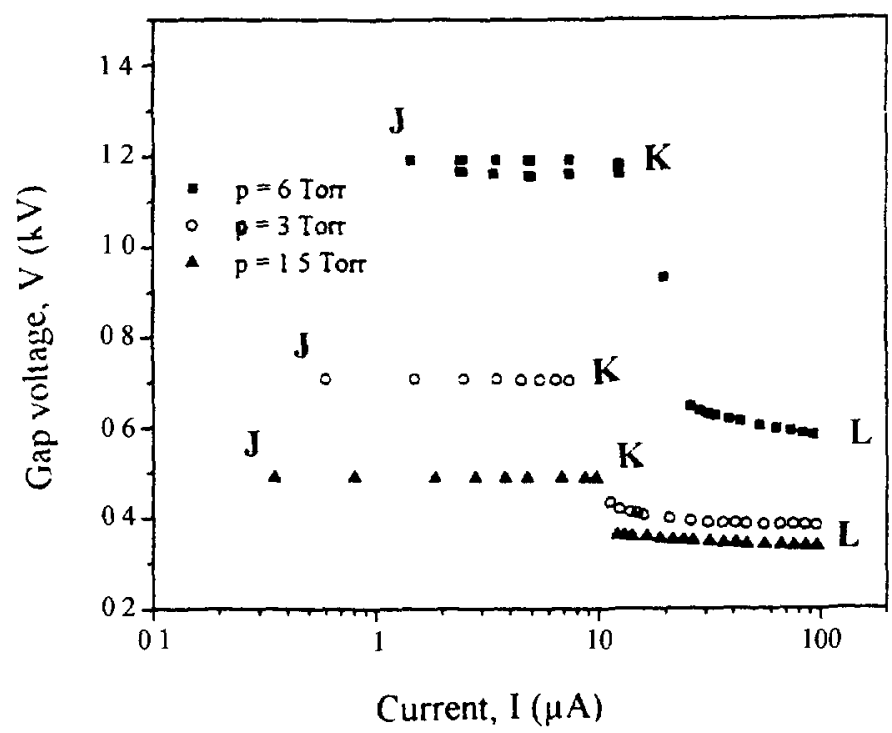

Fig. 7. - Expermental current-voltage characteristics in parallel-plates geometry in nitrogen for $\mathrm{d}=2 \mathrm{~cm}$ and $p=2,4$ and $8 \mathrm{mbar}(1.5,3$ and 6 Torr). These characteristics must be compared to the part JKL of the standard one in Figure [5]

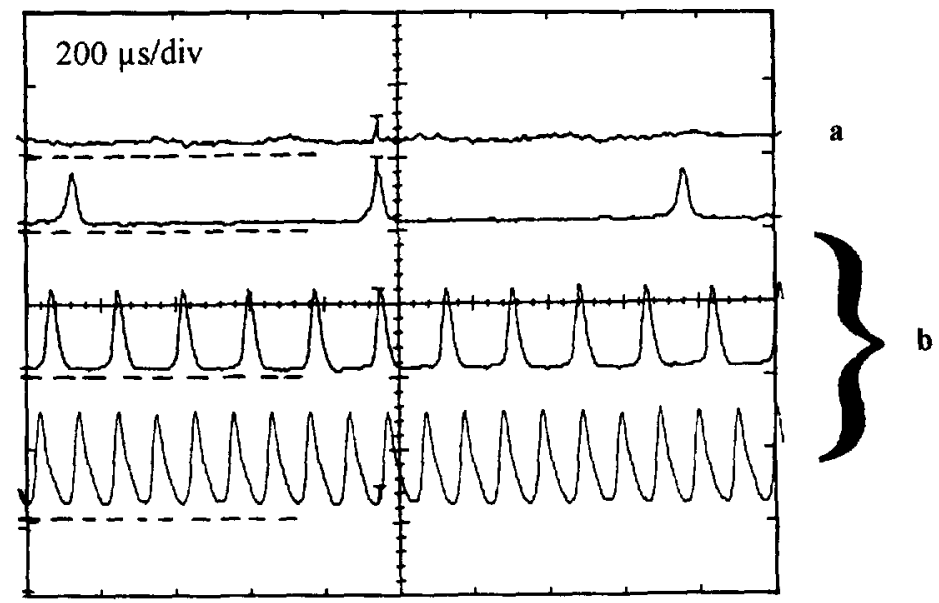

Fig 8. - Nature of the current flowing in the external circuit. Over the part JK (self-sustained dark discharge), a direct current is measured (a). Recurring state composed of transient current impulses superimposed to the direct component are observed in the glow discharge (b) (part KL). a: $5 \mathrm{mV} /$ div.; b: $10 \mathrm{mV} / \mathrm{div}$

$\mathrm{d} V / \mathrm{d} I \approx 0$ and only a direct current is observed. As a first approximation, space charge is then negligible and the increase in current is controlled by the external circuit; thus, $V_{2} \approx V_{3}$. On the part $\mathrm{KL}$, the interelectrode potential is collapsed $(\mathrm{d} V / \mathrm{d} I<0)$ and a recurring state is established with recurring transient current impulses superimposed to the direct component. 


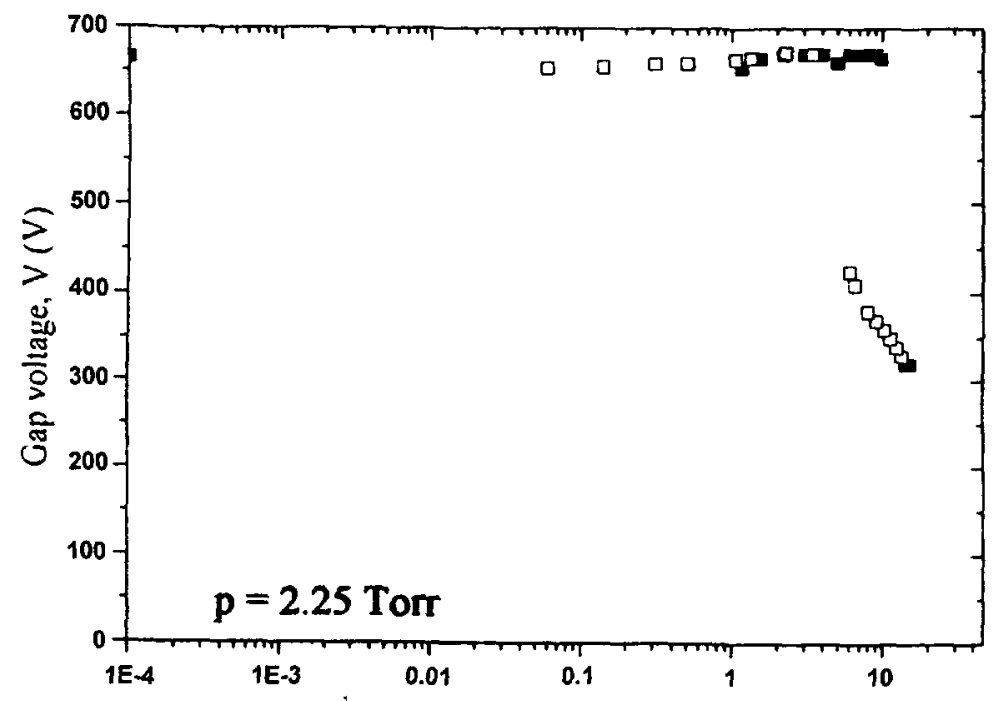

a)

Current. I $(\mu \mathrm{A})$

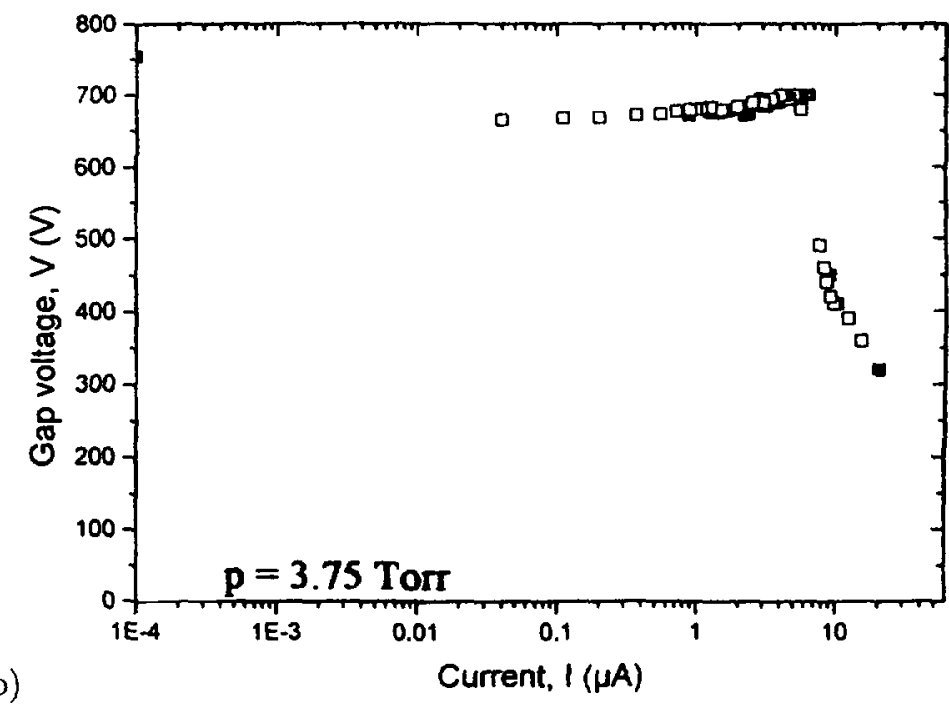

Fig. 9. - Current-voltage characteristics in point-to-plane geometry for $d=1 \mathrm{~cm}$ when pressure lies between 3 and 20 mbar (2.25 and 15 Torr) in nitrogen. $\square \cdot V_{0}$ is increased. $\square . V_{0}$ is decreased

As a conclusion, this $V-I$ characteristic can be compared to the previous ones obtained in parallel-planes geometry (Fig. 7).

For $p=5$ (Fig. 9b), 13.33 (Fig. 9c) and 20 (Fig. 9d) mbar (3.75, 10 and 15 Torr), a positive slope in the part $\mathrm{JK}$ can be observed which grows with pressure. This slope indicates that space charge is no more negligible. Nevertheless, because of the hysteresis, decreasing $V_{0}$ allows to describe a new part of the region $J \mathrm{~K}$ where $I<I_{2}$ and $\mathrm{d} V / \mathrm{d} I \approx 0$. Thus, the voltage $V_{3}$ is related to a space charge free interelectrode gap.

3.4.2. Breakdown in Dry Arr. - Analogue results are obtained in dry air and shown in Figure 10 for $p=1.5$ (Fig. 10a) and 3 mbar (Fig. 10b) (1.125 and 2.25 Torr). Depending on the 


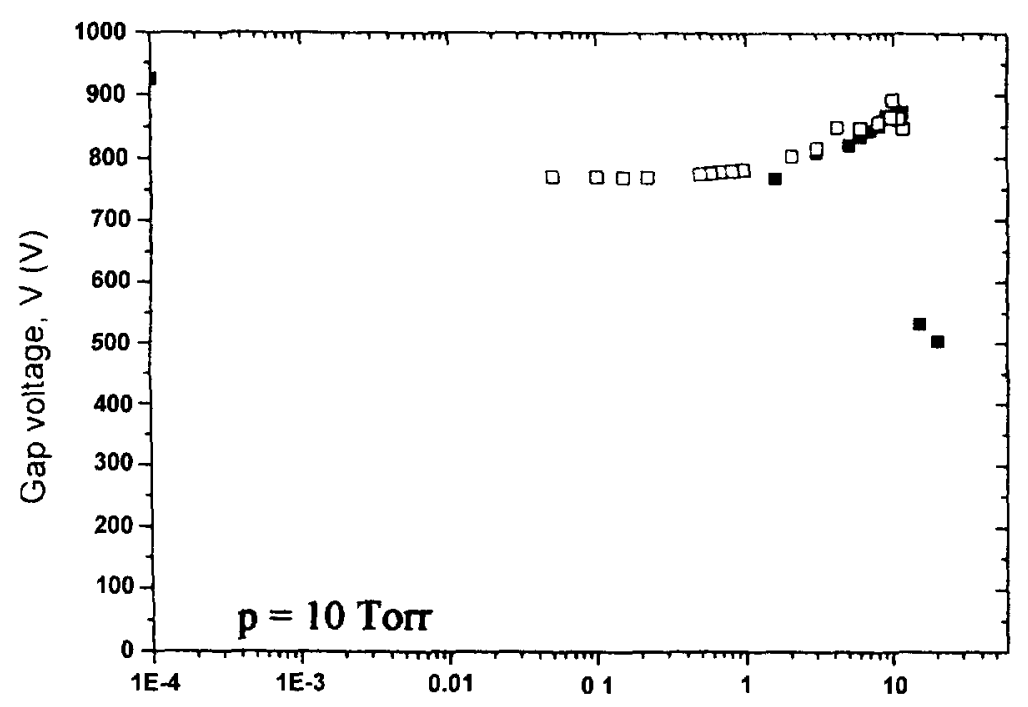

c)

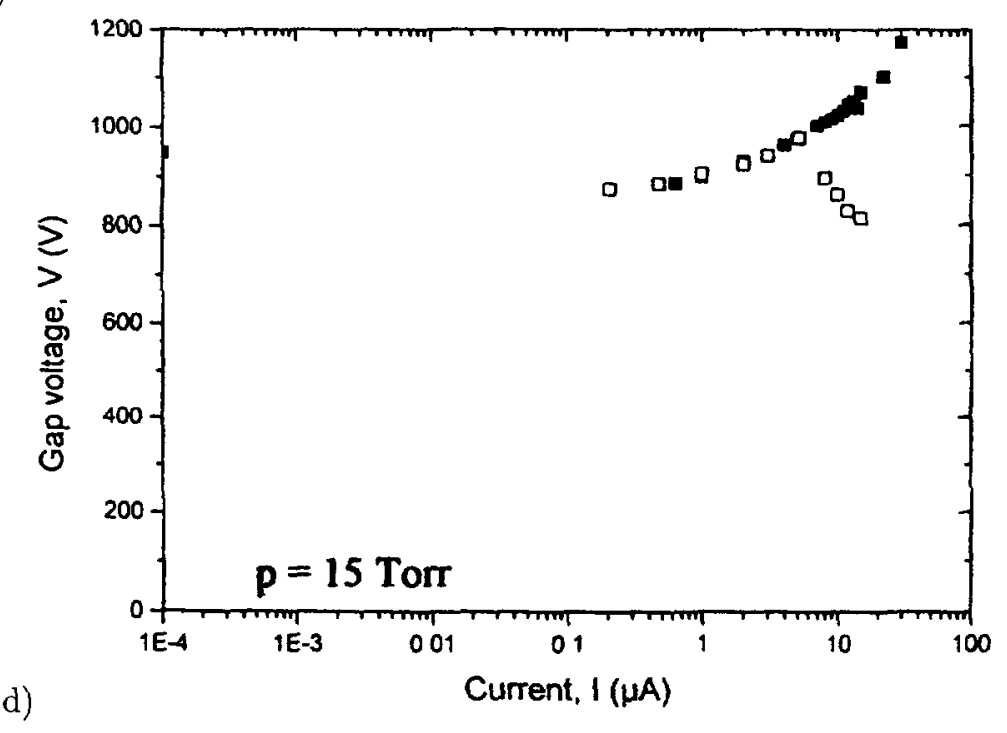

Fig. 9. - (Continued)

pressure, a positive slope in the part JK can be measured, but the hysteresis allows to determine a potential $V_{3}$ corresponding to a space charge free state. as in the nitrogen case.

Nevertheless, a relevant discrepancy between nitrogen and dry air is observed for $p>8$ mbar (6 Torr). As a matter of fact, as it is shown in Figure 11 for $p=20$ mbar (15 Torr), the breakdown in dry air can be associated to the transition to glow discharge, i.e. the part JK corresponding to the dark discharge does not appear on the $V-I$ characteristic One must conclude that the breakdown potential measured is related to the formation of the critical space charge responsible for the transition to glow discharge. 


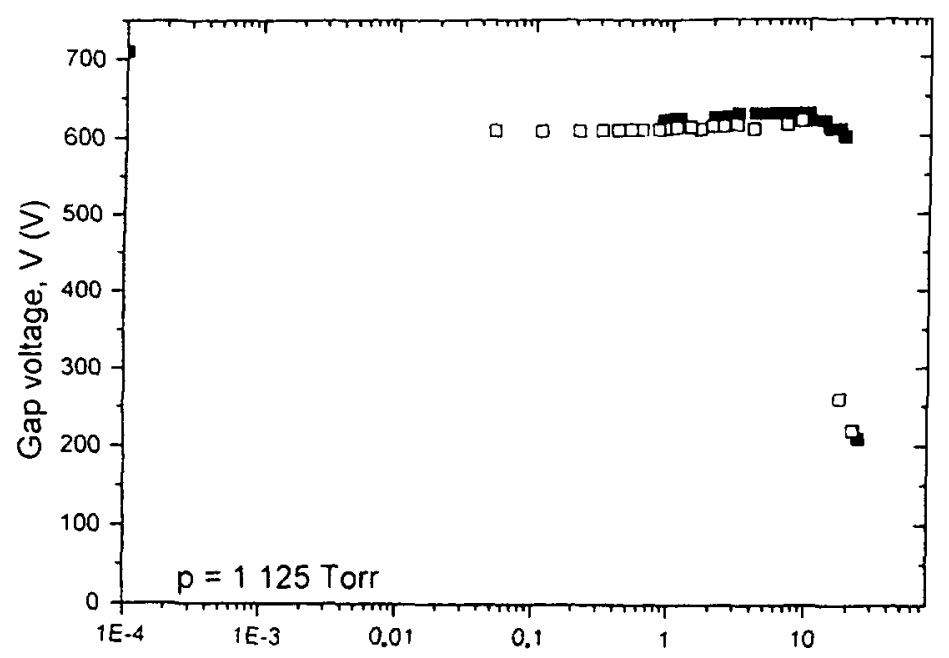

a)

Current, I $(\mu \mathrm{A})$

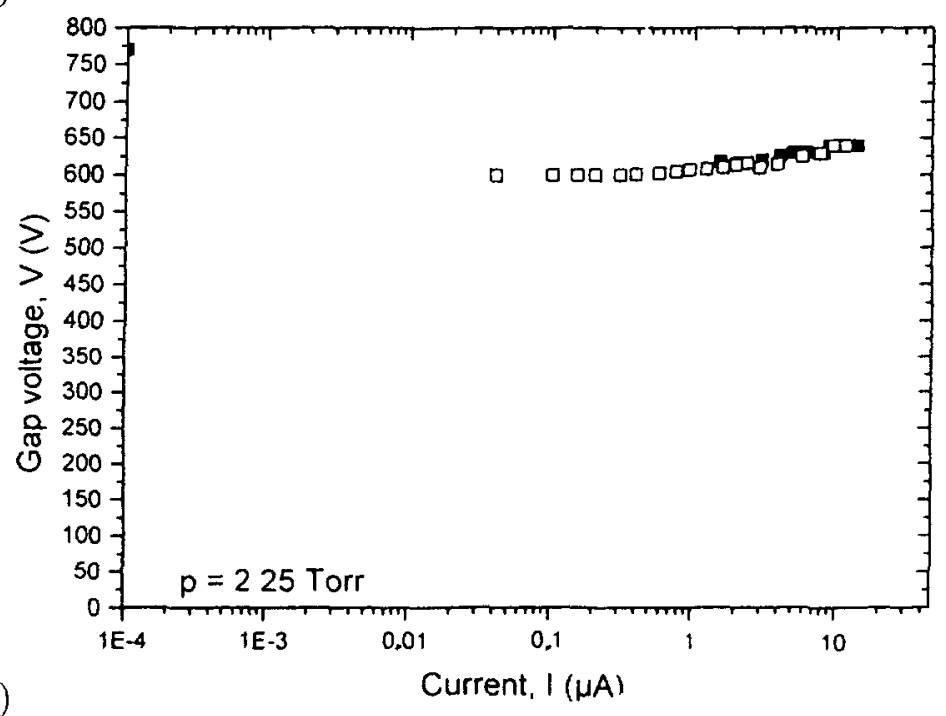

Fig. 10. - Current-voltage characteristics in point-to-plane geometry for $d=1 \mathrm{~cm}$ when $p=1.5$ and 3 mbar (1.125 and 2.25 Torr) in dry air. $\square: V_{0}$ is increased. $\square . V_{0}$ is decreased

3.5. Definition of the Breakdown Voltage and Paschen Curves. - Experimental variations of $V_{1}, V_{2}$ and $V_{3}$ as a function of $p d$ are shown in Figure 12, for nitrogen (Fig. 12a) and dry air (Fig 12b). In order to compare the experimental results with the model which does not take into account the perturbation of the space charge, the potential $V_{3}$, corresponding to the space charge free interelectrode gap, is chosen as the breakdown voltage $V_{\mathrm{B}}$. Moreover, when breakdown is associated to the transition to glow discharge (in dry air), the breakdown voltage is ignored.

A comparison between experimental $V_{\mathrm{B}}=f(p d)$ curves in inhomogeneous and homogeneous fields is presented in Figure 13, for nitrogen (Fig. 13a) and dry air (Fig. 13b). It can be noticed that in both gases, the values of $\left(V_{\mathrm{B}}\right)_{\mathrm{m}}$ and $(p d)_{\mathrm{m}}$ are lowered in parallel-plates geometry. 


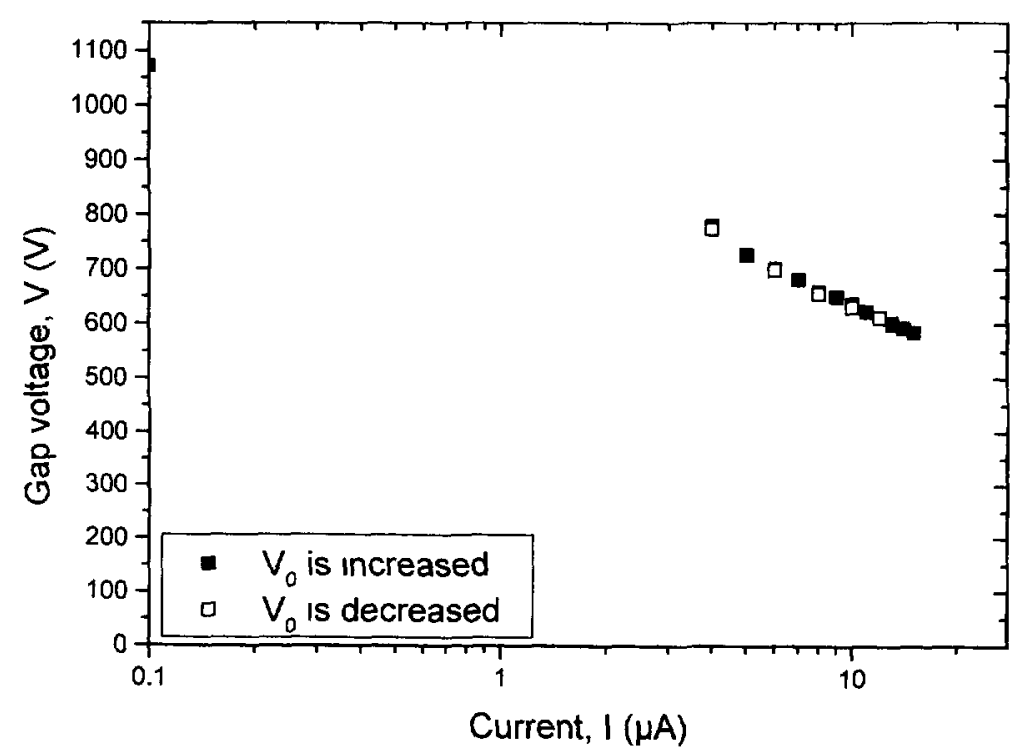

Fig 11. $-V-I$ characteristic in dry air for $p=20 \mathrm{mbar}$ (15 Torr) and $d=1 \mathrm{~cm}$ Electrical breakdown is assoclated to the transition to glow discharge $\square$ : $V_{0}$ is increased. $\square: V_{0}$ is decreased.

Furthermore, the slope of the right-hand side of the Paschen curve is greater in homogeneous field as it has been deduced in the theoretical part of this paper ( $c f$. Figs. 1 and 2).

\section{Comparison Between Theory and Experiments - Discussion}

Before to present a comparison between theory and experiments, it is interesting to do some general remarks concerning the general features of $V_{\mathrm{B}}=f(p d)$ curves. Firstly, one can observe, for given $R / 2 d$ and $p d$ values, an increase of $V_{\mathrm{B}}$ when the secondary ionization coefficient $\gamma$ decreases. This effect can be explained keeping in mind that the self-sustained condition is depending on the ionization mechanisms, the volume of ionization (resulting from the acceleration of primary electrons by the increasing potential) and the effective secondary ionization coefficient being strongly coupled. Secondly, when the ratio $R / 2 d$ decreases, z.e. for a more and more inhomogeneous field, the minimum breakdown potential $\left(V_{\mathrm{B}}\right)_{\mathrm{m}}$ and the corresponding $(p d)_{\mathrm{m}}$ value increase while the slope of the breakdown potential $\mathrm{d}\left(V_{\mathrm{B}}\right) / \mathrm{d}(p d)$ decreases for $(p d)$ values higher than the minimum $(p d)_{\mathrm{m}}$. These general results| will be very useful in the second part of this Section, in order to give an interpretation of the results.

A comparison between theory and experiments is presented in Figure 14 in the point-toplane case when the ratio $R / 2 d=0.05(F(R, d)=3.72)$. The fit' is obtained using for the secondary ionization coefficient $\gamma_{\mathrm{n}}=7.5 \times 10^{-3}$ in nitrogen (Fig. 14a) and $\gamma_{\mathrm{a}}=5.6 \times 10^{-2}$ and $3 \times 10^{-3}$ in air (Fig. 14b). These values are different from those used in a parallel-plates configuration, because $\gamma$ has not the same signification in the point-to-plane case. Although the results are in good agreement, one notices a discrepancy between the experimental and theoretical slopes for $(p d)$ values higher than the minimum $(p d)_{\mathrm{m}}$. This effect can be analysed, keeping in mind that the deviation of experimental Paschen curves from theoretical results (in the parallel-plates configuration) can be interpreted as resulting from uncertainties on the effective secondary ionization coefficient $\gamma[4]$. In this spirit, it is interesting to present a first 


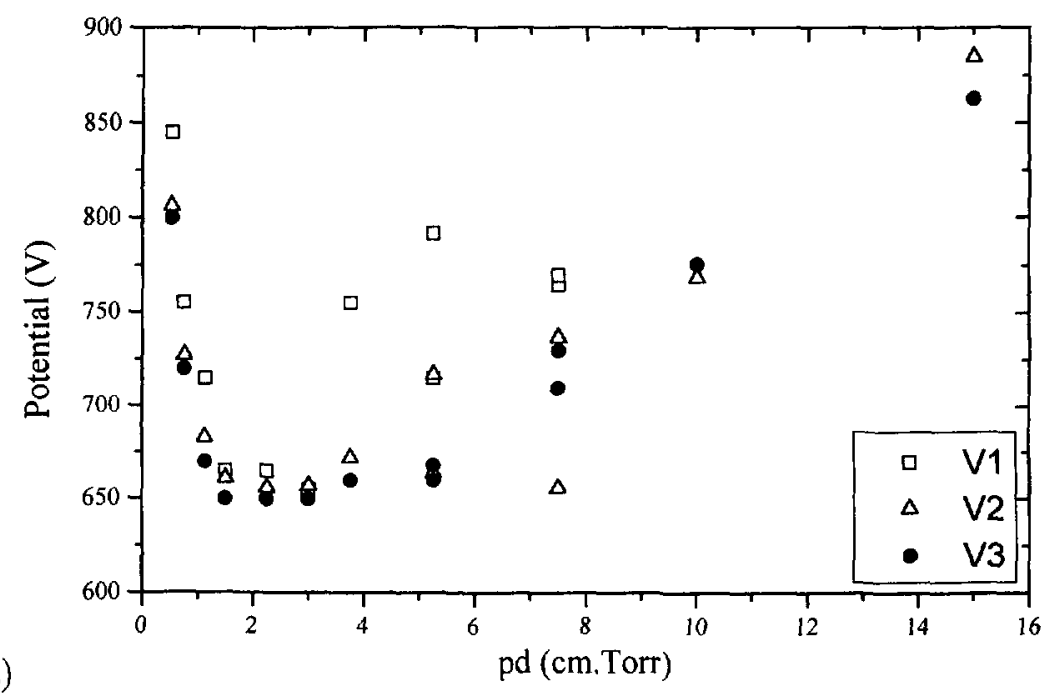

a)

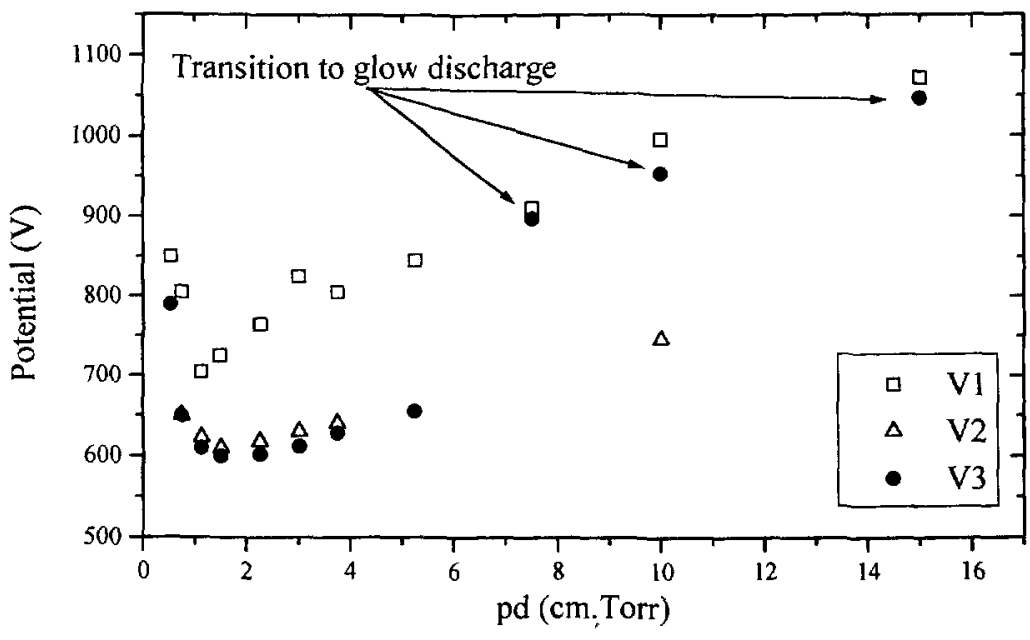

Fig. 12. - a) Experimental variations of $V_{1}, V_{2}$ and $V_{3}$ (see Fig. 6) as a function of $p d$ in nitrogen b) Experimental variations of $V_{1}, V_{2}$ and $V_{3}$ (see Fig 6) as a function of $p d$ in dry air.

evaluation of $\gamma$ as a function of $E / p$ in the parallel-plates case, intraducing experimental $V_{\mathrm{B}}$ and $(p d)$ values in the theoretical Paschen law (7). The results obtained in nitrogen and air are presented in Figures $15 \mathrm{a}$ and $15 \mathrm{~b}$, and it can be observed that $\gamma(E / p)$ is not constant in the range of $E / p$ concerned by these experiments. These results are in agreement with those published in [4] for nitrogen.

At this state of the discussion, it is interesting to notice that, in the point-to-plane configuration, the part of the secondary ionization resulting from ionic bombardment of the plane corresponds to a local reduced field $E(0) / p$ ( i.e. for $z=0$ ) given by (14).

$$
\frac{E(0)}{p}=\frac{V}{p\left(d+\frac{R}{2}\right) \ln \left(1+\frac{2 d}{R}\right)} .
$$

In these conditions, $E(0) / p$ can be determined introducing experimental $V, p$ and $d$ values in 


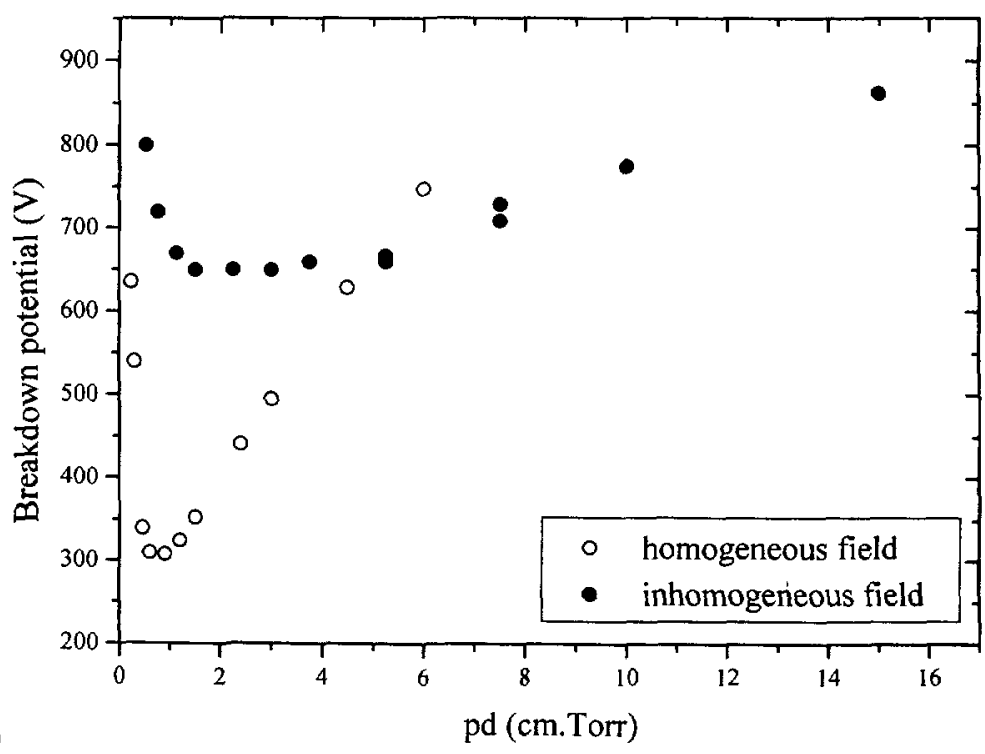

a)

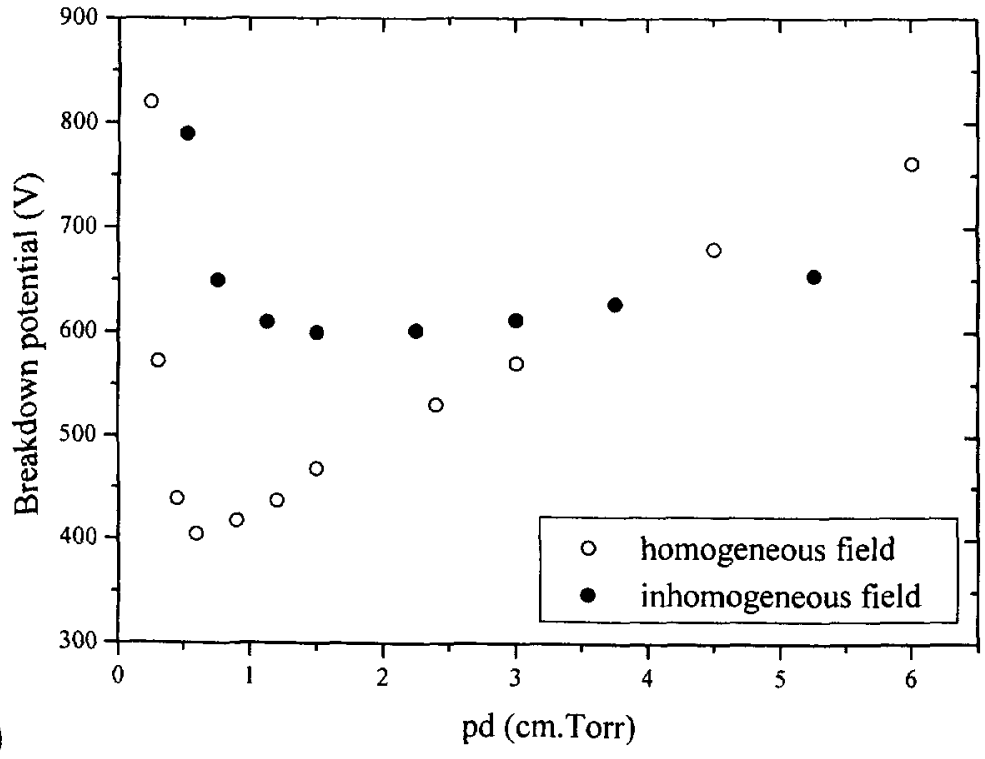

Fig. 13. - a) Comparison between the breakdown potential $V_{B}$ in homogeneous and inhomogeneous fields as a function of $p d$ in nitrogen. b) Comparison between the breakdown potential $V_{\mathrm{B}}$ in homogeneous and inhomogeneous fields as a function of $p d$ in dry air.

(29) for a given $R / 2 d$ ratio. Using the results presented in Figure 13 , at the minimum $\left(V_{\mathrm{B}}\right)_{\mathrm{m}}$, we find $E(0) / p \approx 101.25 \mathrm{~V} \mathrm{~cm}^{-1} \mathrm{mbar}^{-1}\left(135 \mathrm{~V} \mathrm{~cm}{ }^{-1} \mathrm{Torr}^{-1}\right)$ in nitrogen and $E(0) / p \approx$ $93.75 \mathrm{~V} \mathrm{~cm}^{-1}$ mbar $^{-1}\left(125 \mathrm{~V} \mathrm{~cm}^{-1}\right.$ Torr $\left.^{-1}\right)$ in air. For these $E(0) / p$ values, the $\gamma$ values deduced from Figure $15 \mathrm{a}$ and $15 \mathrm{~b}$ in nitrogen and air are closed to the minimum of $\gamma(E / p)$. As a consequence, when $(p d)$ is greater or lower than $(p d)_{\mathrm{m}}, E(0) / p$ decreases or increases and $\gamma$ increases (Figs. 15a and 15b). In these conditions, the constant $\gamma$ value introduced in the theory is lower than the effective $\gamma$ value and the theoretical $V_{\mathrm{B}}$ potentıals are overestimated for a given 


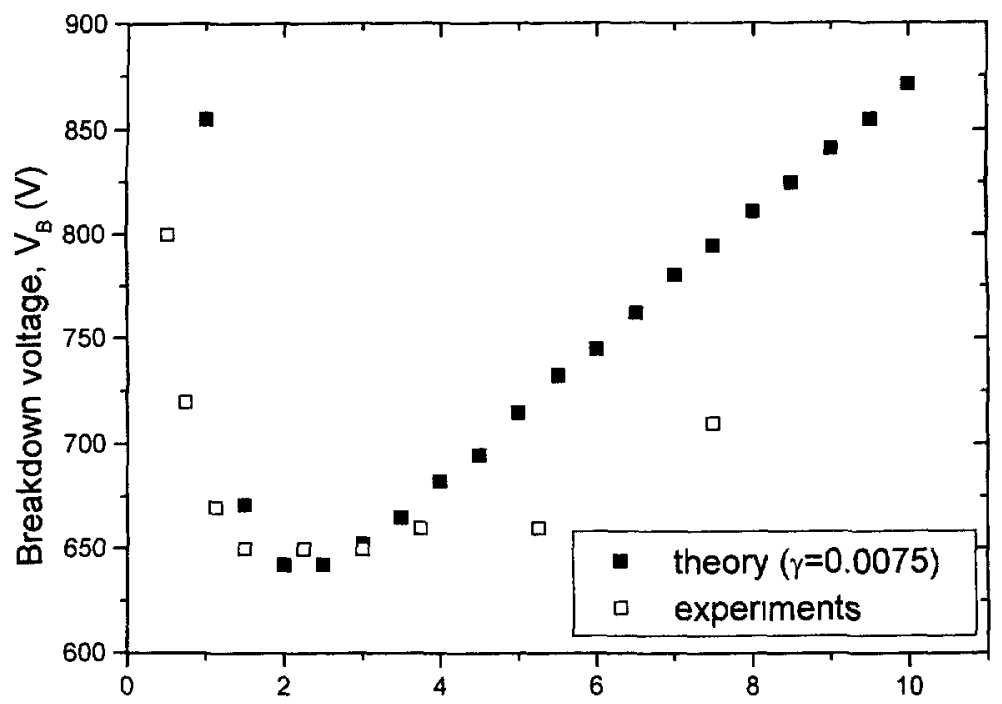

a)

pd (cm.Torr)

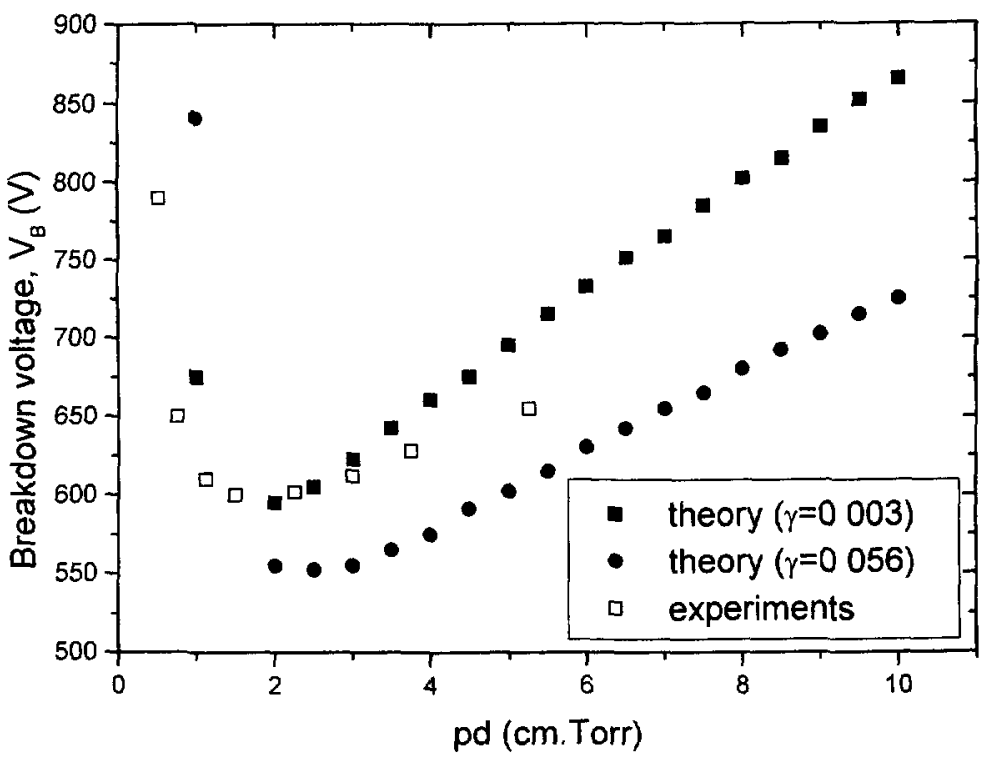

Fig 14 - a) $V_{\mathrm{B}}=f(p d)$ in nitrogen. Comparison between theory and experıments. b) $V_{\mathrm{B}}=f(p d)$ in alr. Comparison between theory and experiments.

$p d$ ( $V_{\mathrm{B}}$ decreases when $\gamma$ increases). This effect can explain a part of the discrepancy between the experimental and theoretical slopes for $(p d)$ values higher than the $(p d)_{\mathrm{m}}$ in Figure 14.

Finally, it is interesting to point out that the first ionization coefficient $\alpha(6)$, depending on the two parameters $A$ and $B$, must be known in a wide range of ratio $E / p$, in order to take care of the non uniformity of the field in the interelectrode gap. 


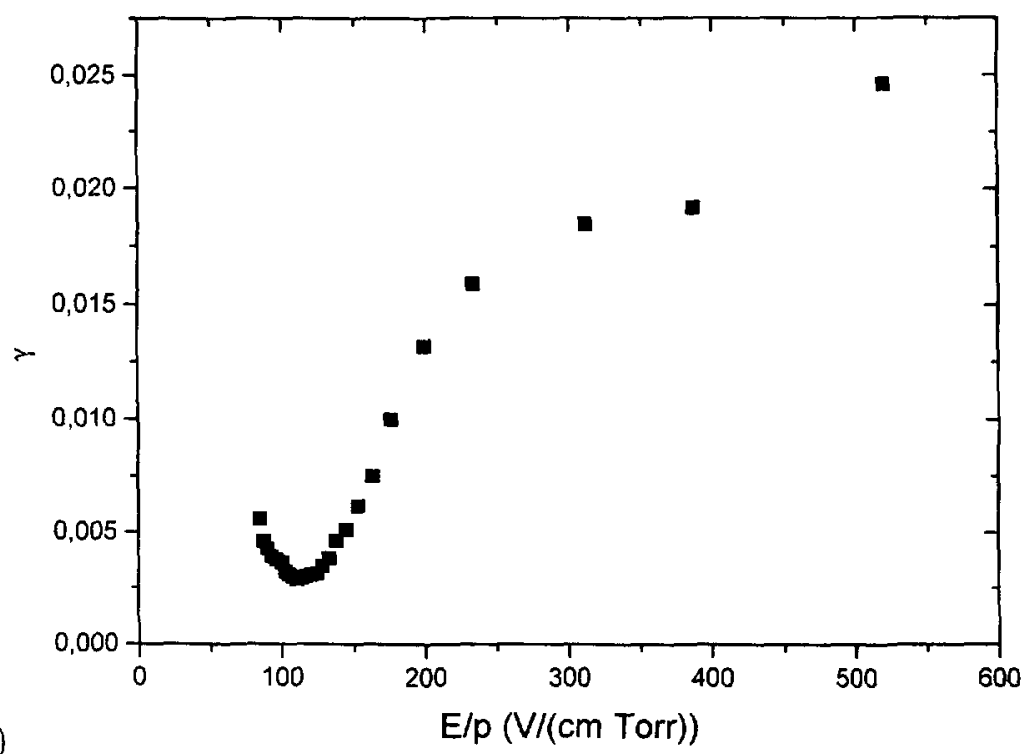

a)

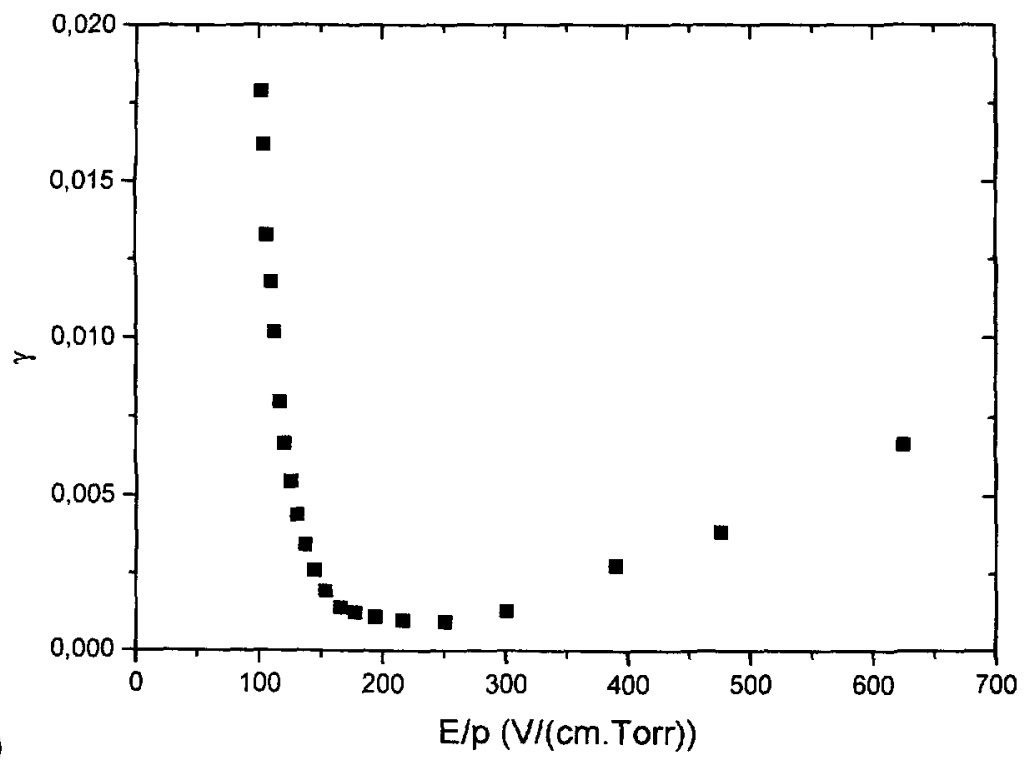

Fig. 15. - a) Secondary ionization coefficient $\gamma$ as a function of the reduced electric field $E / p$ in nitrogen b) Secondary ionization coefficient $\gamma$ as a function of the reduced electric field $E / p$ in air.

\section{Conclusion}

The purpose of this paper was to show that it is possible to deduce $V_{\mathrm{B}}=f(p d)$ curves when the electric field is inhomogeneous (point-to-plane case). This theoretical approach starts from the well-known conditions for a self-sustained discharge in an homogeneous field (parallelplates case), the Paschen curve being the asymptotic limit (radius of curvature $R \approx \infty$ ) of the inhomogeneous field. The comparison between theory and experiment shows a good agreement. the minimum breakdown potential $\left(V_{\mathrm{B}}\right)_{\mathrm{m}}$ and the corresponding $(p d)_{\mathrm{m}}$ value increasing with 
the inhomogeneity of the field. Furthermore, the slope of the breakdown potential decreases for $(p d)$ values higher than the minimum $(p d)_{\mathrm{m}}$. Finally, it is important to notice that the first ionization coefficient $\alpha(6)$ introduced in the theory must be only used in a limited $E / p$ range [4], these theoretical results keeping their validity only in this $V / p d$ range. The determination of the parameters $A$ and $B$ of $(6)$ is very important in order to improve the agreement between theory and experiment, in the homogeneous (Paschen) and inhomogeneous (Paschen-like) case. The dependence of the secondary ionization coefficient $\gamma$ on the ratio $E / p$ is also a crucial problem. These questions are in progress.

\section{Acknowledgments}

Authors wish to thank particularly Dr. M. Yousfi for helpful discussions and theoretical results concerning the first ionization coefficient in nitrogen and air.

\section{References}

[1] Von Hippel A.R., Molecular science and molecular engineering (Technology Press of MIT and John Wiley \& Sons, 1959).

[2] Von Engel A., Ionised gases (Oxford University Press, 1965).

[3] Nasser E., Fundamentals of gaseous ionization and plasma electronics (Wiley-Interscience, 1971).

[4] Raizer Y.P., Gas discharge physics (Springer-Verlag, 1991).

[5] Pedersen A., On the electrical breakdown of gaseous dielectrics, IEEE Transaction on Electrical Insulation 24 (1989) pp. 721-739

[6] Goldman M. and Goldman A., Corona discharges, in Gaseous electronics, M.N. Hirsh and H.J. Oskam, Eds. (Academic Press, 1978) vol. 1, pp. 219-290.

[7] Llewellyn-Jones F., Ionization and breakdown in gases (Methuen, London, 1957).

[8] Franklin R.N., Conf. Ionız. Phen., Uppsala 1 (1960) 164.

[9] Peyrous R., Spyrou N. and Held B., Humidity effects on discharge evolution in a low pressure cold plasma reactor: air, Acta Physica Universitatis Comenuanae, XXXV, 1, (1994) pp. 33-46.

[10] Spyrou N., Peyrous R., Soulem N. and Held B., Why Paschen's law cannot be used in low pressure inhomogeneous fields gas discharges, J. Phys. D. 28 (1995) pp 701-710.

[11] Dutton J., A survey of electron swarm data, J. Phys. Chem. Ref. Data 4 (1975) 577-856.

[12] Yousfi M., Azzi N., Segur P, Gallimberti I. and Stangherlin S., Electron- molecule collision cross sections and electron swarm parameters in some atmospheric gases, report, 1988.

[13] Yousfi M., private communication, 1995.

[14] Sigmond R.S., Corona discharges, in Electrical breakdown of gases, Meek J.M. and Craggs J.D. (Eds.) (John Wiley and Sons, 1978) pp. 319-384.

[15] Held B., Soulem N., Peyrous R. and Spyrou N., Conditions for self-sustained DC corona discharge, 11th International Conference on Gas Discharges and Their Applications, Proceeding, 1 (1995) pp 128-131.

[16] Held B., Coronas and their applications, 11th International Conference on Gas Discharges and Their Applications, Proceeding 2 (1995) pp. 514-526.

[17] Held B., Soulem N., Peyrous R. and Spyrou N., Conditions for a self-sustained DC corona discharge, Trans. IEE of Japan 116A 11 (1996) pp. 925-930.

[18] Druyvestein M.J. and Penning F.M., The mechanism of electrical discharges in gases of low pressure, Rev. of Mod. Phys. 12 (1940) pp. 87-174. 